\title{
Problème de Stefan direct dans un milieu semi-transparent gris
}

\author{
V. Le Dez, F. Yousefian, R. Vaillon, D. Lemonnier et M. Lallemand (*) \\ Laboratoire d'Études Thermiques ( $\left.{ }^{* *}\right)$ ENSMA, 86960 Futuroscope, Poitiers, France
}

(Reçu le 10 juillet 1995, révisé le 6 décembre 1995, accepté le 4 janvier 1996)

PACS.65. - Thermal properties of condensed matter

Résumé. - Le problème direct de la surface mobile est étudié lors du processus de fusion dans un milieu semi-transparent gris monodimensionnel à frontières noires ou transparentes. Par une transformation de Landau, le système est étudié dans le domaine spatio-temporel fixe $[0,1] \times$ $[0, \infty]$ adapté à la résolution du champ de luminances. Dans le problème direct, la position de l'interface, sa vitesse d'évolution ainsi que les champs de températures successifs sont recherchés pour un flux de chaleur incident donné et différentes valeurs des paramètres caractéristiques du couplage conduction-rayonnement. L'étude de la source radiative a été effectuée de deux manières, soit par la méthode des ordonnées discrètes, soit par une méthode de lancer de rayons.

\begin{abstract}
The direct moving boundary problem is studied during the melting process of an one-dimensional gray semi-transparent medium fitted with black or transparent boundaries. By means of a Landau transform, the system is studied in a fixed space-temporal domain $[0,1] \times[0, \infty]$ adapted to the solution of the radiative problem. In the direct problem, the interface location, its melting velocity, as well as the successive temperature profiles are carried out as a function of the incident heat flux on the moving interface and for various values of the radiative-conductive coupling parameters. The radiative source term is performed by means of two different methods, either the discrete ordinates method or the ray tracing one.
\end{abstract}

\section{Introduction}

Le problème de la frontière mobile (ou problème de Stefan) étudie le déplacement de l'interface d'un matériau solide lors d'un changement de phase, sous l'influence d'un flux thermique externe (convectif et/ou radiatif). Il concerne un vaste champ d'applications de phénomènes instationnaires (four à fusion de zone, moulage, soudure, stockage de l'énergie à chaleur latente, évolution des chambres magmatiques de volcans). Principalement développé pour les milieux opaques, il n'a encore été que très peu étudié dans les Milieux Semi-Transparents (MST) (c'està-dire émettant-absorbant) au sein desquels le rayonnement thermique infrarouge participe au transfert de chaleur. De telles situations existent cependant, notamment en croissance cristalline ou encore en thermique spatiale pour les phénomènes d'ablation lorsque le bouclier thermique contient des matériaux semi-transparents. Dans le problème de Stefan direct, on recherche à déterminer la position du front de fusion ou de solidification $S(t)$ en fonction du

(*) Auteur auquel doit être adressée la correspondance (Fax : (33) 49498101 )

(**) URA CNRS 1403

(C) Les Éditions de Physique 1996 
temps sous linfluence des conditions extérieures, ainsi que les distributions de température associées au problème dans les deux phases. Quant au problème de Stefan inverse, qui fait suite à cet article [1], il est relatif à l'estimation de la position d'un front en évolution à partir de données de mesures recueillies en des points accessibles du système. Les mesures peuvent être intrusives (c'est le cas de capteurs posés à l'intérieur du milieu ou sur une interface) ou non (observation de luminances émergeant d'un milieu) ; dans le premier cas que nous traiterons en référence [1], le problème est résolu en coopération avec le modèle direct exposé ici, alors que le second, actuellement à l'étude, ne nécessite pas l'association de ce modèle.

Lorsque le solide présente une semi-transparence, le couplage entre les phénomènes de conduction et de rayonnement entraîne l'établissement de conditions radiatives internes qui peuvent modifier profondément le processus de changement de phase d'une part et, les prévisions sur son évolution d'autre part. En effet, si $\alpha$ représente la diffusivité thermique et $S_{0}$ l'épaisseur du milieu, le phénomène de conduction a comme échelle de temps caractéristique $t_{\mathrm{C}}=\frac{S_{0}^{2}}{\alpha}$ alors que dans un MST de coefficient d'absorption $\kappa$ et de nombre de Planck $N_{\mathrm{P}}$. s'introduit une échelle de temps radiatif $t_{\mathrm{R}}=\frac{N_{\mathrm{P}}}{\alpha \kappa^{2}}$ qui peut différer de $t_{\mathrm{C}}$ de plusieurs ordres de grandeur.

L'étude de l'influence de la semi-transparence dans le problème de Stefan a été abordée la première fois par Habib [2] puis reprise par Abrams et Viskanta [3] pour la solidification des cristaux. Chan et al. [4] ont proposé un modèle de changement de phase où coexistent le solide et le liquide semi-transparents. Enfin, le groupe de Purdue University a porté son attention sur les phénomènes de rayonnement et de fusion de la composition dans les fours de verrerie [5].

Au paragraphe 2, nous explicitons le modèle géométrique, les hypothèses retenues ainsi que la formulation relative au problème de Stefan direct. Le paragraphe 3 présente les bases des méthodes directes permettant de résoudre le problème de Stefan par différences finies en insistant sur la méthode du front fixe introduite par Landau [6] qui, par une simple transformation des coordonnées, permet de développer un schéma implicite à pas de temps et d'espace réduits constants. Dans les MST, le rayonnement agit comme une source volumique qui modifie les distributions de température et de flux qui s'établiraient en son absence : ce couplage rayonnement-conduction instationnaire est étudié au paragraphe 4 dans la géométrie monodimensionnelle réduite en considérant que le milieu est gris et les parois limitant le système noires.

Néanmoins, la méthode développée s'appliquerait également pour un milieu non gris dont le coefficient d'absorption est, soit uniquement spectralement dépendant, soit éventuellement aussi thermo-dépendant : ceci peut en effet survenir dans certains cristaux où la dépendance du spectre d'absorption est relativement bien connue en fonction de la température [7] et où ce phénomène peut avoir une influence, notamment au voisinage des zones en fusion. l'augmentation de température déplaçant en général le front d'absorption vers des longueurs d'onde plus basses [8]. Cependant, dans le cadre de cette étude, nous nous limiterons au cas d'un milieu gris non thermo-dépendant, et dans ce but, la Méthode des Ordonnées Discrètes (MOD), ainsi que celle du Lancer des Rayons (LR), est mise en œuvre pour déterminer la densité de puissance de la source radiative.

\section{Le problème de Stefan direct dans un MST gris}

2.1. LE MODÈLE PHYSIQUE. - On considère un système monodimensionnel constitué d une phase solide semi-transparente initialement à la température ambiante et d'épaisseur $S(t=0)=S_{0}$. Une des faces est soumise à un échelon de flux thermique total fixe $F$. Dans ces conditions, l'évolution de la température dans le solide s'effectue en deux temps; en un 
premier temps la température de la face irradiée $T\left(S_{0}, t\right)$ s'accroît jusqu'à ce qu'elle atteigne la température de fusion $T_{\mathrm{f}}$; en un second temps, l'interface se déplace : sa position instantanée est alors $S(t)$, en restant à la température de changement de phase franche fixée. On suppose que les conditions aux limites thermiques à la paroi froide (en $x=0$ ) sont déterminées par les échanges convectifs et radiatifs entre cette paroi et l'environnement, à la température ambiante constante $T_{a}$, (coefficient de transfert de chaleur $h$ ). En ce qui concerne l'autre phase (liquide ou gazeuse) nous simplifierons le problème en admettant qu'elle est évacuée au fur et à mesure de sa production en y négligeant donc la convection. Dans le problème de Stefan direct, la position de l'interface solide-liquide $S(t)$ du milieu semi-transparent est à déterminer sous l'effet du flux de chaleur externe constant ainsi que d'autres paramètres comme la température de la paroi fixe, les profils de température dans la phase solide $T(x, t)$ et la vitesse de fusion, et ce, en fonction des conditions de couplage interne, elles-mêmes évolutives, entre le rayonnement et la conduction.

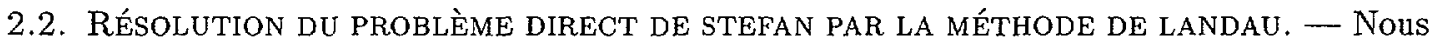
utiliserons la méthode de front-fixe de Landau [6] développée ultérieurement par Furzeland [9] dont l'intérêt est d'offrir un cadre spatial fixe bien adapté à la description du champ radiatif, que ce soit par la méthode des ordonnées discrètes ou celle du lancer de rayons.

\subsection{Formulation mathématique du PRoblème de STEFAn diREct en Couplage} RAYONNEMENT-CONDUCTION. - Le mur solide plan parallèle d'épaisseur instantanée $S(t)$ est considéré du point de vue radiatif comme gris, c'est-à-dire que son coefficient d'absorption $\kappa_{\lambda}=\kappa$ reste constant sur tout le spectre infrarouge. Le produit $\tau^{0}(t)=\kappa S(t)$ représente l'épaisseur optique de ce mur ; elle sera variable tout au long du processus de changement de phase. La paroi externe et l'interface seront, soit toutes deux opaques et noires, soit l'une transparente et l'autre opaque, soit toutes deux transparentes. Sous l'influence du flux externe se développent, au sein de la matière, des échanges combinés entre le mode de transfert par rayonnement et celui par conduction, entre le milieu et ses frontières et entre les parois lorsque celles-ci sont émissives. La figure 1 représente schématiquement les apports des différents $f$ ux de chaleur associés aux processus.

L'équation de la chaleur instationnaire dans la phase solide semi-transparente s'écrit en géométrie monodimensionnelle

$$
\frac{\partial T}{\partial t}(x, t)=\alpha \frac{\partial^{2} T}{\partial x^{2}}(x, t)-\frac{1}{\rho_{\mathrm{v}} c} \frac{\partial q^{\mathrm{r}}}{\partial x} \quad \text { pour } 0 \leq x \leq S(t) \quad t>0
$$

correspondant à la superposition locale des mécanismes de transfert par diffusion et par rayonnement, représenté par le terme de source, $\rho_{\mathrm{v}}$ étant la masse volumique, $c$ la chaleur massique et la diffusivité $\alpha=K / \rho_{\mathrm{v}} c$, où $K$ est la conductivité thermique.

Dans un milieu semi-transparent gris, la divergence du flux radiatif et le flux radiatif ont respectivement la forme suivante [10]

$$
\begin{aligned}
\operatorname{div} \mathbf{q}^{\mathbf{r}} & =\frac{\partial q^{\mathbf{r}}}{\partial x}=\kappa\left[4 n_{\mathbf{r}}^{2} \sigma T^{4}-\int_{\Omega=4 \pi} L(\boldsymbol{\Omega}) \mathrm{d} \Omega\right] \\
\mathbf{q}^{\mathbf{r}} & =\int_{\Omega=4 \pi} \boldsymbol{\Omega} L(\boldsymbol{\Omega}) \mathrm{d} \Omega
\end{aligned}
$$

où $L(\boldsymbol{\Omega})$ désigne la luminance directionnelle (direction $\boldsymbol{\Omega}$ ) totale (intégrée sur le spectre), $\sigma$ la constante de Stefan et $n_{\mathrm{r}}$ l'indice de réfraction du milieu. 

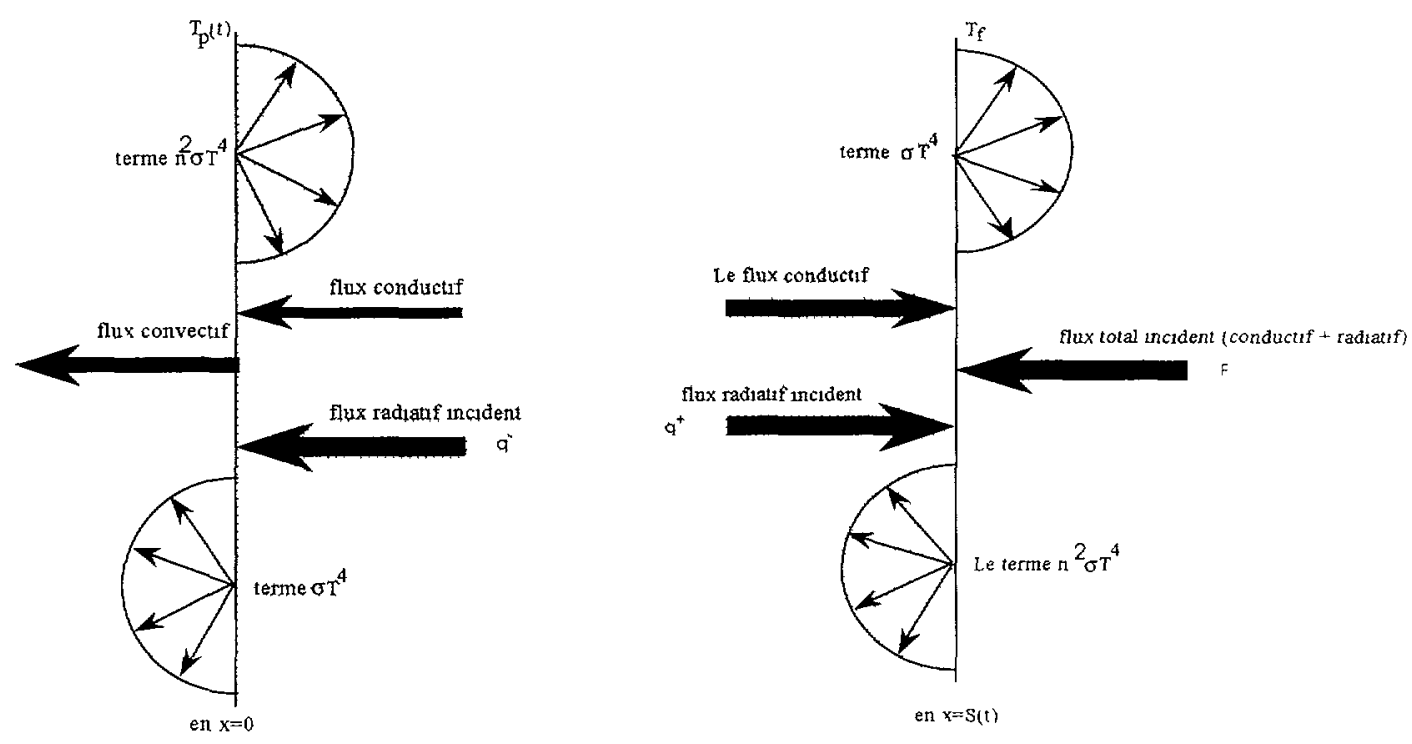

Fig. 1. - Les conditions aux limites thermiques en flux du mur plan de MST.

[Thermal boundary conditions of the plane STM wall.]

Lorsque le milieu est gris et ses frontières noires, la condition à la paroi froide s écrit

$$
-K^{r} \frac{\partial T}{\partial x}+q^{-}=h\left(T_{\mathrm{p}}-T_{\mathrm{a}}\right)+\left(n_{\mathrm{r}}^{2}+1\right) \sigma T_{\mathrm{p}}^{4}-\sigma T_{\mathrm{a}}^{4} \quad \text { en } \quad x=0 \quad t>0
$$

et celle à la paroi chaude

$$
K^{2} \frac{\partial T}{\partial x}-F-q^{+}\left(n_{\mathrm{t}}^{2}+1\right) \sigma T_{\mathrm{f}}^{4}-\sigma T_{\mathrm{a}}^{4}=\rho \mathrm{L} \frac{\partial S}{\partial t}(t) \quad \text { en } \quad x=S(t) \quad t>0
$$

enfin à l'interface d'impact on a :

$$
T(x . t)=T_{\mathrm{f}} \quad \text { en } \quad x=S(t) \quad t>0
$$

Dans les relations (4) et (5) les termes $q^{-}, q^{+}$désignent les flux radiatifs incidents, respectivement, à la paroi froide et à la paroi chaude, alors que les termes $\left(n_{\mathrm{r}}^{2}+1\right) \sigma T^{4}$ correspondent aux contributions de l'émission des parois noires de part et d'autre de la frontière. On a

$$
\begin{aligned}
& q^{-}=\int_{|\Omega n|<0} L(x=0, \Omega)|\Omega n| \mathrm{d} \Omega \\
& q^{+}=\int_{|\Omega n|>0} L[x=S(t), \Omega]|\Omega n| \mathrm{d} \Omega
\end{aligned}
$$

Quant au terme de droite de l'équation (5), L étant la chaleur latente de fusion. il représente la quantité de chaleur absorbée par unité de temps pour fondre la paroi. 


\section{Résolution par la méthode implicite de Landau}

3.1. LES ÉQUATIONS TRANSFORMÉEs. - Par le changement de variables

$$
\chi=\frac{x}{S\left(t^{*}\right)} \quad t^{*}=\frac{\alpha}{S_{0}^{2}} t \quad \tau^{0}\left(t^{*}\right)=\kappa S\left(t^{*}\right) \quad \theta=\frac{T}{T_{\mathrm{f}}} \quad 0 \leq \chi \leq 1
$$

et en introduisant les caractéristiques optiques et de couplage $\tau^{0}$ et $N_{\mathrm{P}}=\frac{\kappa K}{4 n_{\mathrm{r}}^{2} \sigma T_{\mathrm{f}}^{3}}$ (ce dernier représentant la proportion du flux conductif par rapport au flux radiatif dans les transferts du MST) l'équation de transfert de chaleur dans la phase solide devient

$$
\frac{\partial \theta}{\partial t^{*}}=\chi \frac{\dot{\tau}^{0}}{\tau^{0}} \frac{\partial \theta}{\partial \chi}+\frac{\left(\tau_{0}^{0}\right)^{2}}{\left(\tau^{0}\right)^{2}} \frac{\partial^{2} \theta}{\partial \chi^{2}}-\frac{\left(\tau_{0}^{0}\right)^{2}}{N_{\mathrm{p}}}\left(\theta^{4}-\frac{G}{4 \sigma n_{\mathrm{r}}^{2} T_{\mathrm{f}}^{4}}\right) \quad \text { pour } 0 \leq \chi \leq 1 \text { et } t>0
$$

où $G$ désigne l'énergie totale incidente apparaissant dans le second terme de la divergence du flux radiatif $(2)$, soit

$$
G=\int_{\Omega=4 \pi} L(\Omega) \mathrm{d} \Omega
$$

$\tau_{0}^{0}$ l'épaisseur optique initiale, et $\dot{\tau}^{0}=\frac{\mathrm{d} \tau^{0}}{\mathrm{~d} t^{*}}$ la dérivée par rapport au temps de l'épaisseur optique.

Remarque sur l'adimensionnement en temps. - Nous avons privilégié le temps caractéristique conductif $t_{\mathrm{C}}$ par rapport au temps caractéristique radiatif $t_{\mathrm{R}}$. Le rapport de ces deux temps donnant $\frac{t_{\mathrm{C}}}{t_{\mathrm{R}}}=\frac{\left(\tau_{0}^{0}\right)^{2}}{N_{\mathrm{P}}}$, proportionnel à $\kappa$, il apparaît que dans le cas d'un milieu très faiblement diffusant, pour une température de fusion relativement élevée, les deux temps caractéristiques sont du même ordre de grandeur uniquement pour des coefficients d'absorption extrêmement faibles, qui n'ont pas été retenus dans cette étude. Au contraire, notre choix s'est porté sur des milieux dont les absorptions sont suffisamment élevées, proches des milieux denses, pour lesquels le temps caractéristique conductif est très supérieur au temps caractéristique radiatif : ceci explique le choix de notre adimensionnement.

À l'interface mobile, on a la condition thermique

$$
\begin{aligned}
& \frac{\partial \theta}{\partial \chi}-\tau^{0} \Phi+\frac{\tau^{0}}{4 n_{\mathrm{r}}^{2} N_{\mathrm{P}}}\left[\left(n_{\mathrm{r}}^{2}+1\right) \theta_{\mathrm{f}}^{4}-\theta_{\mathrm{a}}^{4}-4 Q^{+}\right]=\frac{\tau^{0} \dot{\tau}^{0}}{\left(\tau_{0}^{0}\right)^{2} \mathrm{St}} \quad \text { en } \chi=1 \quad t>0 \\
& \theta\left(\chi, t^{\times}\right)=\theta_{\mathrm{f}}=1 \quad \text { en } \quad \chi=1 \quad t>0
\end{aligned}
$$

où le flux imposé réduit est $\Phi=F / \kappa K T_{\mathrm{f}}$. Enfin la condition à l'interface fixe (opaque)

$$
\left.-\frac{\partial \theta}{\partial \chi}=\operatorname{Bi}\left(\theta_{\mathrm{p}}-\theta_{\mathrm{a}}\right)+\frac{\tau^{0}}{4 n_{\mathrm{r}}^{2} N_{\mathrm{p}}}\left[n_{\mathrm{r}}^{2}+1\right) \theta_{\mathrm{p}}^{4}-\theta_{\mathrm{a}}^{4}-4 Q^{-}\right] \text {en } \chi=0 \quad t>0
$$

Dans les équations (10a et $10 \mathrm{~b}) Q^{+}$et $Q^{-}$sont les flux radiatifs incidents aux parois, adimensionnés, générés par le milieu $\left(Q^{ \pm}=q^{ \pm} / 4 \sigma T_{\mathrm{f}}^{4}\right)$.

Le nombre de Stefan $\mathrm{St}=c T_{\mathrm{f}} / \mathrm{L}$ introduit dans l'équation (10a) représente l'importance relative de la chaleur sensible par rapport à la chaleur latente. Quant au nombre de Biot, $\mathrm{Bi}=\frac{h S}{K}$, il représente l'importance relative du flux convectif par rapport au flux conductif. 


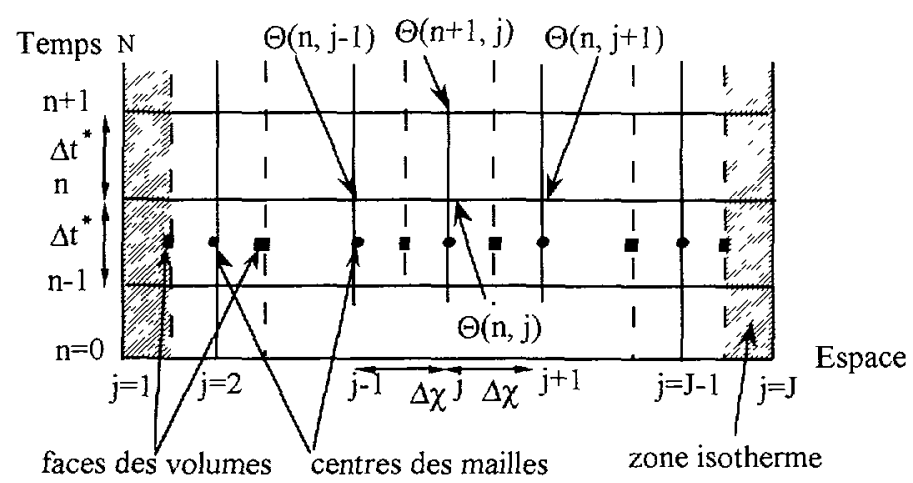

Fig. 2. - Maillage spatio-temporel du mur.

[Space-temporal meshing of the wall.]

3.2. LE MAILLAGE SPATIO-TEMPOREL UTILISÉ. - La transformation de Landau conduisant à $0 \leq \chi \leq 1$ permet d'utiliser un pas de temps constant associé à un nombre constant de mailles. Le domaine de calcul $(\chi, t) \in[0,1] \times[0, \infty]$ est subdivisé de manière uniforme en un nombre $J$ de nœuds et un nombre $N$ de pas de temps, ce dernier dépendant de l'épaisseur initiale du mur et de la physique du problème ; le maillage spatio-temporel $(n, \jmath)\left(n=1,2, \quad \Lambda^{r} ; \jmath=1\right.$, $2, . ., J)$ associé est représenté sur la figure 2 .

Les équations ont été discrétisées par une méthode de différences finies explicite en temps, associée à des différences centrées pour les dérivées d'espace. L'équation (8) devient

$$
\begin{aligned}
& \left(\tau^{02}\right)^{n+1, k} \frac{\theta_{3}^{n+1, k+1}-\theta_{3}^{n}}{\Delta t^{*}}=\left[\left(\dot{\tau}^{0}\right)^{n+1, k}\left(\tau^{0}\right)^{n+1, k} \lambda_{3} \frac{\left(\theta_{3+1}^{n+1}-\theta_{j-1}^{n+1}\right)^{k+1}}{2 \Delta \lambda}\right. \\
& \left.+\left(\tau_{0}^{0}\right)^{2} \frac{\left(\theta_{\jmath+1}^{n+1}-2 \theta_{\jmath}^{n+1}+\theta_{\jmath-1}^{n+1}\right)^{k+1}}{\Delta \chi^{2}}\right] \\
& +\frac{\left(\tau_{0}^{0}\right)\left(\tau^{0}\right)^{n+1, k}}{N_{\mathrm{P}}}\left\{\frac{G\left(\theta_{\jmath}^{n}\right)}{4 n_{\mathrm{r}}^{2} \sigma T_{\mathrm{f}}^{4}}-\left[4\left(\theta_{3}^{n, k}\right)^{3} \theta_{\jmath}^{n+1, k+1}-3\left(\theta_{\jmath}^{n, k}\right)^{4}\right]\right\}
\end{aligned}
$$

où

$$
\left(\dot{\tau}^{0}\right)^{n+1, k}=\frac{\left(\tau^{0}\right)^{n+1, k}-\left(\tau^{0}\right)^{n}}{\Delta t^{\times}}
$$

Pour résoudre l'équation (11) il est nécessaire de connaître la valeur de l'épaisseur optique $\tau^{0}$ et de sa vitesse $\dot{\tau}^{0}$ aux instants $(n+1)$; celles-ci sont obtenues à l'aide d'un calcul intermédiaire portant sur l'indice $k$ (qui sera explicité plus bas, cf. Éqs. (29), (30), (31)) imposé par le bilan énergétique sur la paroi mobile. La discrétisation des conditions aux limites mène aux relations supplémentaires :

- en $x=0$ :

$$
\begin{array}{r}
-\frac{\theta_{2}^{n+1}-\theta_{1}^{n+1}}{\Delta \chi}=\mathrm{Bi}^{n}\left(\theta_{1}^{n+1}-\theta_{\mathrm{a}}\right)+\frac{\left(\tau^{0}\right)^{n}}{4 n_{\mathrm{r}}^{2} N_{\mathrm{P}}}\left\{\left(n_{\mathrm{r}}^{2}+1\right)\left[4\left(\theta_{1}^{n}\right)^{3} \theta_{1}^{n+1}-3\left(\theta_{1}^{n}\right)^{4}\right]-\theta_{\mathrm{a}}^{4}-4 \mathrm{Q}^{-n}\right\} \\
t>0
\end{array}
$$




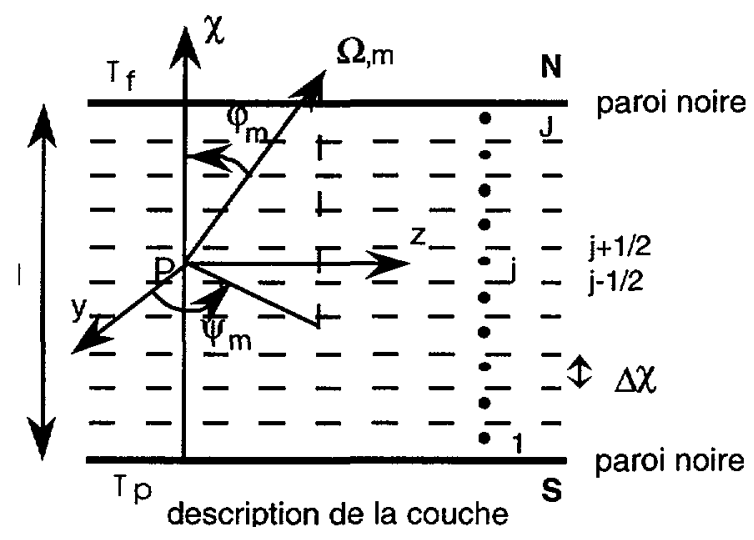

Fig. 3. - Système d'axes de la méthode des ordonnées discrètes.

[Axis system of the discrete ordinates method.]

- en $\chi=1$ :

$$
\theta_{J}^{n+1}=\theta_{\mathrm{f}} \quad t>0
$$

Le système linéaire tridiagonal constitué par les équations (11), (12) et (13) ainsi obtenu est classiquement résolu en températures par l'algorithme de Thomas.

\section{Les sources radiatives}

4.1. Par la Méthode des Ordonnées Discrètes (MOD). - La Méthode des Ordonnées Discrètes permet la détermination du champ des luminances directionnelles lorsqu'on connaît le champ thermique. Nous considérons un ensemble de directions $\boldsymbol{\Omega}_{m}$ décrites chacune par deux angles $\varphi_{m}$ (angle polaire définissant une latitude) et $\psi_{m}$ (azimut) (Fig. 3).

Les termes intégrés sur les angles solides $(2,3)$ sont calculés à partir de formules de quadratures portant sur $M$ directions

$$
\begin{aligned}
G & =\int_{\Omega=4 \pi} L(\boldsymbol{\Omega}) \mathrm{d} \Omega=\sum_{m=1}^{M} w_{m} L^{m} \quad \text { (énergie incidente) } \\
\mathrm{q}^{\mathrm{r}} & =\int_{\Omega=4 \pi} \boldsymbol{\Omega} L(\boldsymbol{\Omega}) \mathrm{d} \Omega=\sum_{m=1}^{M} w_{m} L^{m} \boldsymbol{\Omega}_{m} \quad \text { (densité de flux) }
\end{aligned}
$$

où $\boldsymbol{\Omega}_{m}$ décrit l'ensemble des $M$ directions discrètes considérées (abscisses de la quadrature), les $w_{m}$ sont les poids de la quadrature et les $L^{m}$ sont les luminances totales dans les directions discrètes $\boldsymbol{\Omega}_{m}$ associées.

Puisque le milieu est infini suivant les axes $y$ et $z$, la luminance est indépendante de $\psi$. Par conséquent, nous étudierons les luminances par latitude et les quadratures se réduisent à

$$
\begin{aligned}
& G=2 \pi \int_{0}^{\pi} L(\varphi) \sin \varphi \mathrm{d} \varphi=2 \pi \int_{-1}^{1} L(\mu) \mathrm{d} \mu=2 \pi \sum_{n=1}^{N} \omega_{n} L^{n} \\
& q^{\mathrm{r}}=2 \pi \int_{0}^{\pi} L(\varphi) \cos \varphi \sin \varphi \mathrm{d} \varphi=2 \pi \int_{-1}^{1} \mu L(\mu) \mathrm{d} \mu=2 \pi \sum_{n=1}^{N} \omega_{n} \mu_{n} L^{n}
\end{aligned}
$$


où $\mu=\cos \varphi_{n}$ est le cosinus directeur définissant la latitude $n$. $\hat{\gamma}^{\prime}$ est le nombre de latitudes de l'ensemble des directions considéré et les $\omega_{n}$ sont les poids de la nouvelle quadrature.

Le problème central en rayonnement repose sur la détermination du champ des luminances directionnelles dans tout le domaine spatial par résolution de l'Équation de Transfert Radiatif (ÉTR) que l'on peut écrire pour chaque latitude particulière $\mu_{n}$

$$
\frac{\mu_{n}}{S} \frac{\mathrm{d} L^{n}}{\mathrm{~d} \chi}+\kappa L^{n}=\kappa n_{\mathrm{r}}^{2} L^{0}(T)
$$

où $L_{0}(T)$ est la luminance intégrée du corps noir à la température $T$ telle que

$$
L^{0}(T)=\sigma_{\mathrm{B}} \frac{T^{4}}{\pi}
$$

Pour les surfaces noires, les conditions aux limites radiatives associées sont

$$
\begin{aligned}
& L^{n}(\chi=0)=L^{0}\left(T_{\mathrm{p}}\right) \quad(n=1 . N) \\
& L^{n}(\chi=1)=L^{0}\left(T_{\mathrm{f}}\right) \quad\left(n=1, N^{\top}\right)
\end{aligned}
$$

L'intégration de l'ÉTR (18) sur un volume de contrôle $\Delta \lambda \times 1 \times 1$ conduit à la relation

$$
\mu_{n}\left(L_{\mathrm{N}}^{n}-L_{\mathrm{S}}^{n}\right)+\kappa S \Delta \chi L_{\mathrm{P}}^{n}=\mathrm{S}_{\mathrm{P}} S \Delta \chi
$$

où $L_{\mathrm{P}}^{n}, L_{\mathrm{N}}^{n}$ et $L_{\mathrm{S}}^{n}$ sont respectivement les luminances au centre, au nord et au sud de la maille et

$$
\mathrm{S}_{\mathrm{P}}=\kappa n_{\mathrm{r}}^{2} \sigma_{\mathrm{B}} \frac{T^{4}}{\pi}
$$

Le principe de détermination des luminances $L_{\mathrm{P}}^{n}$ dans tout le domaine est alors le suivant : lorsque $\mu_{n}>0$, la luminance de la face sud $L_{S}^{n}$ étant connue par les conditions aux limites (20), la luminance $L_{\mathrm{P}}^{n}$ se calcule d'après (22) en fonction de $L_{\mathrm{S}}^{n}$, mais aussi de $L_{\mathrm{N}}^{n}$ qui est inconnue. Pour lever cette indétermination, on procède par interpolation des luminances $L_{\mathrm{N}}^{n}$, $L_{\mathrm{S}}^{n}$ et $L_{\mathrm{P}}^{n}$

$$
L_{\mathrm{P}}^{n}=\alpha L_{\mathrm{N}}^{n}+(1-\alpha) L_{\mathrm{S}}^{n}
$$

$\alpha$ étant un paramètre d'interpolation $(0<a<1)$. 


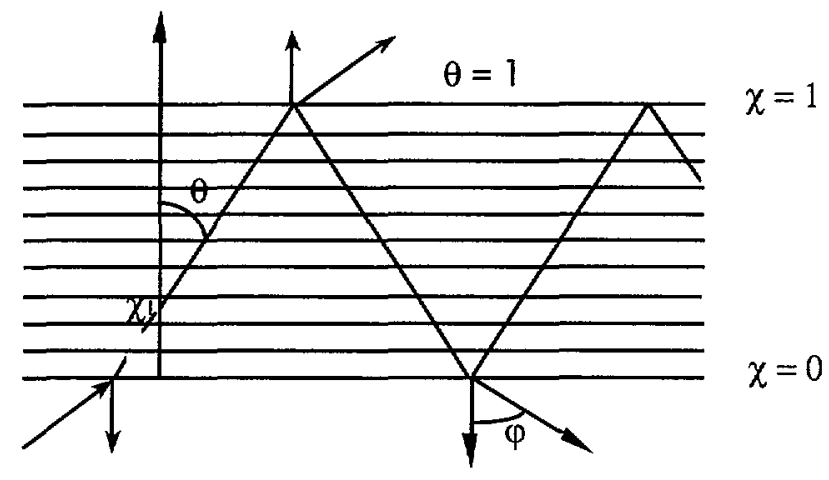

Fig. 4. - Le trajet des rayons dans le mur.

[Ray path in the wall.]

$\grave{A}$ partir des équations (22) et (24), l'expression de la luminance $L_{\mathrm{P}}^{n}$ au centre de la maille devient

$$
L_{\mathrm{P}}^{n}=\frac{\alpha \mathrm{S}_{\mathrm{P}} S \Delta \chi+\mu_{n} L_{\mathrm{S}}^{n}}{\alpha \kappa S \Delta \chi+\mu_{n}}
$$

La luminance $L_{\mathrm{N}}^{n}$ est calculée à partir de la relation (24). Le procédé est renouvelé à la maille suivante et se répète jusqu'à obtention du profil des luminances directionnelles.

Lorsque $\mu_{n}<0$, la même méthode est employée mais en s'appuyant cette fois sur $L_{\mathrm{N}}^{n}$ et l'équation (21). Nous avons choisi un coefficient d'interpolation $\alpha=0,5$. Ce schéma, communément dénommé diamant, est celui qui mène aux résultats les plus précis.

L'utilisation de formules de quadratures dans les relations (16) et (17) nécessite de choisir les abscisses et les poids de celles-ci. Nous avons adopté une quadrature de Gauss.

Nous avons utilisé cette méthode en raison de sa simplicité de mise en ceuvre dans une géométrie particulièrement peu complexe ; bien que mal adaptée au traitement des réflexions spéculaires aux interfaces, elle permet de traiter facilement le cas de surfaces noires ou à réflexion diffuse. Cependant, la possibilité de réflexions spéculaires sur des surfaces pouvant être transparentes dans le cas de cristaux, nous a conduits également à calculer la source radiative à l'aide de la méthode du lancer des rayons, particulièrement bien appropriée à l'étude de ce cas. En outre, cette technique permet la détermination aisée d'indicatrices directionnelles d'émission apparente pouvant être utilisées à la résolution du problème inverse lorsqu'on ne dispose pas de mesures de températures internes ou de surface par des capteurs.

4.2. LA MÉThODE DU LANCER DE RAYONS (LR). - Cette méthode purement géométrique, qui autorise également la détermination du champ des luminances directionnelles en un point du milieu pour un champ thermique connu après calcul des trajectoires réelles, est particulièrement bien adaptée au cas des réflexions spéculaires aux interfaces [11]. Les expressions de la source radiative interne et des flux aux interfaces qui en résultent, sont, pour le cas particulier de la plaque plane, purement analytiques, faisant intervenir explicitement, outre le champ global thermique et les phénomènes de réflexion aux deux interfaces, l'épaisseur optique variable au cours du temps. Nous avons appliqué cette technique au cas d'un mur dont l'interface froide est soit opaque, soit transparente : dans cette dernière situation, les facteurs de réflexion polarisés parallèlement et perpendiculairement à l'interface sont directionnels, gouvernés par les lois de Descartes (les facteurs de réflexion étant donnés par les lois de Fresnel pour des 
constantes optiques données), et la méthode permet d'obtenir les indicatrices d'émission apparentes directionnelles qui subiront des modifications de forme et d'intensité dues aux variations temporelles du champ thermique et à l'évolution du front de fusion (Fig. 4).

Compte tenu de l'invariance géométrique du système par rotation, les repérages directionnels ne font intervenir qu'un paramètre angulaire, $\mu=\cos \theta$.

Dans la méthode LR, le terme source (9) de l'équation (8) devient sous forme adimensionnée [12] :

$$
\begin{aligned}
\frac{G}{2 \sigma T_{\mathrm{f}}^{4}}= & \int_{\mu=0}^{1}\left\{\frac { \mathrm { e } ^ { - \frac { \tau ^ { 0 } } { \mu } \chi } + \rho \mathrm { e } ^ { - \frac { \tau ^ { 0 } } { \mu } ( 2 - \chi ) } } { 1 - \rho \overline { \rho } \mathrm { e } ^ { - 2 \frac { \tau ^ { 0 } } { \mu } } } \left[\delta(1-\bar{\rho}) \theta_{\mathrm{a}}^{4}+(1-\delta) \alpha \theta_{\mathrm{S}_{1}}^{4}\right.\right. \\
& \left.\left.+\bar{\rho} \frac{\tau^{0}}{\mu} \int_{\chi=0}^{1} \theta^{4}\left(\chi, t^{*}\right) \mathrm{e}^{-\frac{\tau^{0}}{\mu} \chi} \mathrm{d} \chi\right]\right\} \mathrm{d} \mu \\
& +\int_{\mu=0}^{1}\left\{\frac { \mathrm { e } ^ { - \frac { \tau ^ { 0 } } { \mu } ( 1 - \chi ) } + \overline { \rho } \mathrm { e } ^ { - \frac { \tau ^ { 0 } } { \mu } ( 1 + \chi ) } } { 1 - \rho \overline { \rho } \mathrm { e } ^ { - 2 \frac { \tau ^ { 0 } } { \mu } } } \left[\left(1-\delta^{\prime}\right) \varepsilon+\delta^{\prime}(1-\rho)\left(\theta_{\mathrm{a}}^{4}+\frac{F}{\sigma}\right)\right.\right. \\
& \left.\left.+\rho \frac{\tau^{0}}{\mu} \int_{\chi=0}^{1} \theta^{4}\left(\chi, t^{\times}\right) \mathrm{e}^{-\frac{\tau^{0}}{\mu}(1-\chi)} \mathrm{d} \chi\right]\right\} \mathrm{d} \mu \\
& +\tau^{0} \int_{\mu=0}^{1}\left[\int_{\chi^{\prime}=0}^{\chi} \theta^{4}\left(\chi^{\prime}, t^{\times}\right) \mathrm{e}^{-\frac{\tau^{0}}{\mu}\left(\chi-\chi^{\prime}\right)} \mathrm{d} \chi^{\prime}+\int_{\chi^{\prime}=\chi}^{1} \theta^{4}\left(\chi, t^{\times}\right) \mathrm{e}^{-\frac{\tau^{0}}{\mu}\left(\chi^{\prime}-\chi\right)} \mathrm{d} \chi^{\prime}\right] \frac{\mathrm{d} \mu}{\mu}
\end{aligned}
$$

suivant que les interfaces sont opaques $\left(\delta=0\right.$ pour l'interface fixe, $\delta^{\prime}=0$ pour l'interface mobile) ou transparentes $\left(\delta=1\right.$, respectivement $\left.\delta^{\prime}=1\right) ; \rho$ et $\bar{\rho}$ sont les facteurs de réflexion respectivement à l'interface d'impact et à l'interface froide. Les intégrales dans l'expression de $G$ peuvent être discrétisées de sorte que le terme source de l'équation (8) peut se mettre sous la forme [12]:

$$
\frac{1}{N_{\mathrm{P}}}\left(\theta_{\imath}^{4}-\frac{\mathrm{G}_{\imath}}{4 \sigma T_{\mathrm{f}}^{4}}\right)=\sum_{k=1}^{J} B_{k}^{2} \theta_{k}^{4}
$$

où les coefficients $B_{k}^{2}$ variables au cours du temps font intervenir les distances parcourues par le rayonnement entre le nœud $\imath$ et les nœuds $k$. L'intégrale directionnelle en $\mu$, analogue à celle apparaissant dans l'équation (14), est calculée numériquement à l'aide d'une quadrature de Gauss à 16 points, puis introduite dans le système général (11). Les termes de flux radiatif apparaissant aux conditions limites sont traités de la même manière.

\section{Méthode de résolution numérique et résultats}

õ.1. LE SYSTÈME TRIDIAGONAL ET LE SCHÉMA ITÉRATIF. -- Lorsque le rayonnement est traité par la MOD l'équation (11). se met sous la forme

$$
\left[\begin{array}{cccccc}
b_{1} & c_{1} & & & & 0 \\
& a_{3} & b_{J} & c_{3} & & \\
& & & & & \\
0 & & & & a_{J} & b_{J}
\end{array}\right]^{n+1, k}\left[\begin{array}{c}
\theta_{1} \\
\theta_{J} \\
\cdot \\
\theta_{J}
\end{array}\right]^{n+1, k+1}=\left[\begin{array}{c}
d_{1} \\
d_{3} \\
\cdot \\
d_{J}
\end{array}\right]^{n, k}
$$

Pour résoudre le système tridiagonal (28) à chaque pas de temps, il est nécessaire de connaître la valeur de $\tau^{0}\left(t^{\star}\right)$ et de sa dérivée temporelle $\dot{\tau}^{0}$. Ces valeurs dépendent elles-mêmes des 
solutions de la température dans les deux phases par l'intermédiaire de l'équation de bilan d'énergie (10a) exprimée en $\chi=1$

$$
\frac{\partial \theta^{n+1, k+1}}{\partial \chi}-\left(\tau^{0}\right)^{n+1, k+1} \Phi+\frac{\left(\tau^{0}\right)^{n+1, k+1}}{4 n_{\mathrm{r}}^{2} N_{\mathrm{P}}}\left[\left(n_{\mathrm{r}}^{2}+1\right) \theta_{\mathrm{f}}^{4}-\theta_{\mathrm{a}}^{4}-4 Q^{t^{n}}\right]=\frac{\left(\tau^{0}\right)^{n+1, k+1}\left(\dot{\tau}^{0}\right)^{n+1, k+1}}{\left(\tau_{0}^{0}\right)^{2} \mathrm{St}}
$$

Dans la phase solide, la dérivée de la température à la paroi peut être approchée par

$$
\frac{\partial \theta^{n+1, k+1}}{\partial \chi}=\frac{\theta_{J}^{n+1, k+1}-\theta_{J-1}^{n+1, k+1}}{\Delta \chi}+\theta(\Delta \chi)
$$

dans ces conditions, on obtient une nouvelle valeur de la vitesse $\dot{\tau}^{0}$, ce qui donne pour $\tau^{0}$

$$
\left(\tau^{0}\right)^{n+1, k+1}=\left(\tau^{0}\right)^{n}+\left(\dot{\tau}^{0}\right)^{n+1, k+1} \Delta t^{*}
$$

5.2. RÉSultats. - Les conditions physiques choisies sont les suivantes : épaisseur initiale du mur $S(t=0)=0,1$ ou $1 \mathrm{~m}$, sa température de fusion est $T_{\mathrm{f}}=1000 \mathrm{~K}, T_{\mathrm{a}}=300 \mathrm{~K}$, le coefficient d'échange sur la paroi fixe, $h$, correspond à une loi de convection naturelle pour un mur vertical, à coefficients de transport variables. Le mur semi-transparent est soumis à un échelon de flux $F=150 \mathrm{~kW} / \mathrm{m}^{2}$. Le nombre de Stefan est constant et égal à 1 . Les propriétés thermo-physiques sont $\rho_{\mathrm{v}}=2000 \mathrm{~kg} / \mathrm{m}^{3}, \mathrm{~L}=500 \mathrm{~kJ} / \mathrm{K}$, diffusivité $\alpha$ égale soit à $10^{-5}$ soit à $10^{-6} \mathrm{~m}^{2} / \mathrm{s}$. Le coefficient d'absorption gris du MST $\kappa$ varie entre 0,1 et $1000 \mathrm{~m}^{-1}$, l'indice de réfraction du milieu est constant, de valeur $n_{\mathrm{r}}=1,5$. Les deux parois sont, soit noires et opaques, soit totalement transparentes.

L'évolution des profils de température est obtenue par résolution du système (28) avant la fusion pour l'interface fixe en posant $\dot{\tau}^{0}=0$ et (28) à partir de la fusion de l'interface mobile. Pour la surface mobile un critère d'arrêt des calculs en épaisseur à été choisi égal à $1 \mathrm{~mm}$.

5.2.1. Cas d'un MST à frontières opaques noires. - On a reporté sur les figures $5 \mathrm{a}, \mathrm{b}$, les profils de température adimensionnés à différents temps pour le mur opaque et pour un MST dont le coefficient d'absorption vaut $\kappa=0,1 \mathrm{~m}^{-1}$ pour une même diffusivité $\left(\alpha=10^{-6}\right)$, tous deux à frontières noires. Les résultats obtenus, tant par la méthode des ordonnées discrètes que par la méthode du lancer des rayons pour le calcul de la source radiative interne, sont pratiquement identiques pour les cas étudiés.

On peut constater en comparant le cas opaque et celui du transfert couplé :

1) qu'à partir du moment où le mur commence à fondre, la température de la paroi fixe du MST croît plus rapidement que dans le mur opaque, ceci étant dî pour une part au profil de départ qui pénètre plus profondément dans le MST que dans le mur opaque,

2) l'élévation en température de la paroi froide s'effectue dans le cas semi-transparent d'autant plus vite que le milieu est moins absorbant, excepté aux derniers instants. On remarque également que lorsque le milieu est clair apparaît un minimum dans le profil de température correspondant au réchauffement de la paroi fixe directement par le flux radiatif émis par la paroi noire mobile.

L'évolution de la température de paroi fixe noire des différents murs a été reportée sur la figure 6 ; aux petits temps le comportement est très différent selon que les épaisseurs optiques initiales sont élevées ou faibles, les comportements aux grands temps correspondants s'inversant selon ces valeurs. 

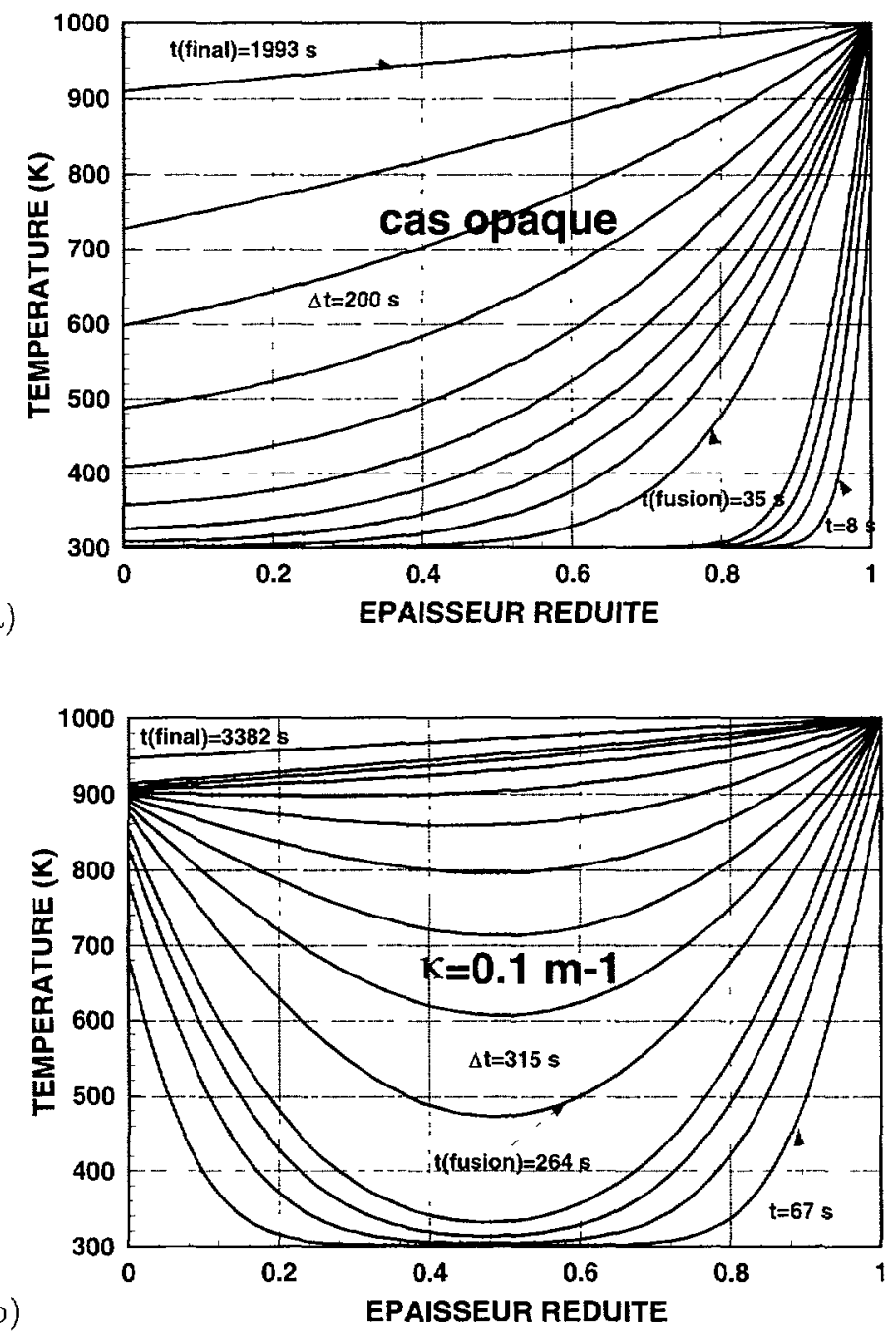

Fig. 5. - Profils de température dans le mur à parois noires à différents instants, a) cas opaque. b) mur gris de coefficient d'absorption $0,1 \mathrm{~m}^{-1}$

[Temperature profiles of the wall with black boundaries for several times, (a) opaque case, (b) gray wall case of absorption coefficient of $0,1 \mathrm{~m}^{-1}$ ]

La figure 7 représente l'évolution temporelle comparée de l'épaisseur de six types de murs de MST ; on constate que pour les caractéristiques optiques et thermodynamiques particulières mentionnées, à flux incident identique, les murs de MST fondent plus lentement que les murs opaques, les cas extrêmes demandant sensiblement 1,5 fois plus de temps. La vitesse de fusion des six murs en fonction du temps est représentée sur la figure 8 ; elle augmente rapidement aux premiers instants, passe par un maximum d'autant plus élevé que le mur est optiquement épais, les courbes d'évolution étant presque confondues pour les milieux faiblement absorbants. 


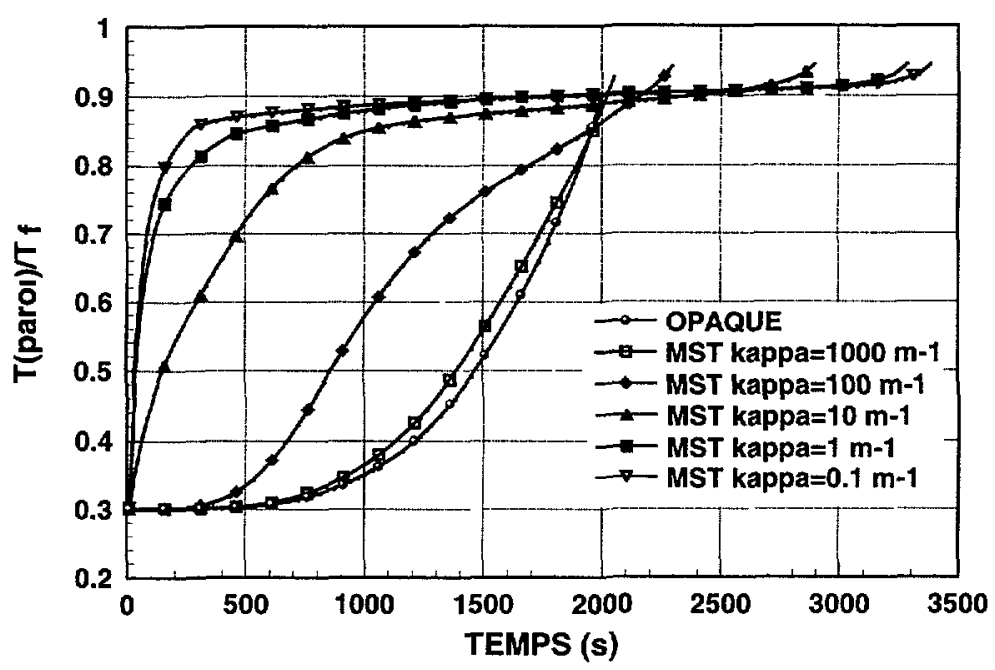

Fig. 6. - Évolution en fonction du temps de la température d'une paroi fixe noire d'un mur plan de $10 \mathrm{~cm}$ pour différents coefficients d'absorption.

[Evolution as a function of time of the temperature at the fixed black wall of a $10 \mathrm{~cm}$ plane wall for several absorption coefficients.]

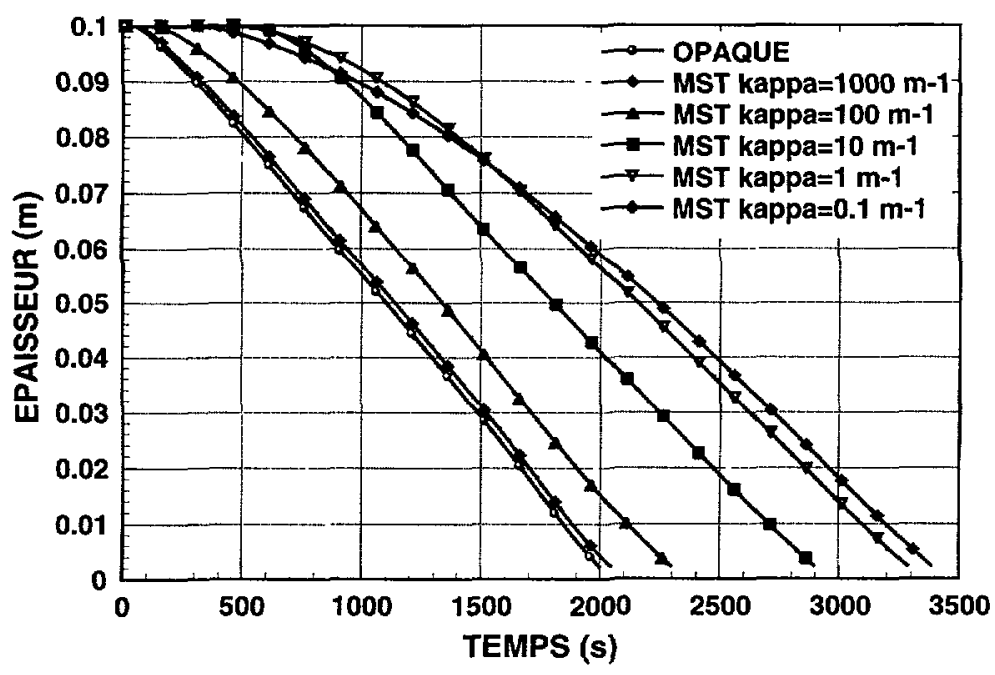

Fig. 7. - Évolution en fonction du temps de l'épaisseur d'un mur plan gris de $10 \mathrm{~cm}$ pour différents coefficients d'absorption.

[Evolution as a function of time of the thickness of a $10 \mathrm{~cm}$ gray wall for several absorption coefficients.]

5.2.2. Cas d'un MST à frontières transparentes. - Ces résultats ont été obtenus par la méthode du lancer des rayons. L'épaisseur du mur a été choisie égale à $0,1 \mathrm{~m}$ et sa diffusivité thermique a pour valeur $10^{-6} \mathrm{~m}^{2} / \mathrm{s}$; le coefficient d'absorption a été fixé à $10 \mathrm{~m}^{-1}$ et le flux incident est $F=120 \mathrm{~kW} / \mathrm{m}^{2}, T_{\mathrm{f}}$ restant fixé à $1000 \mathrm{~K}$. 


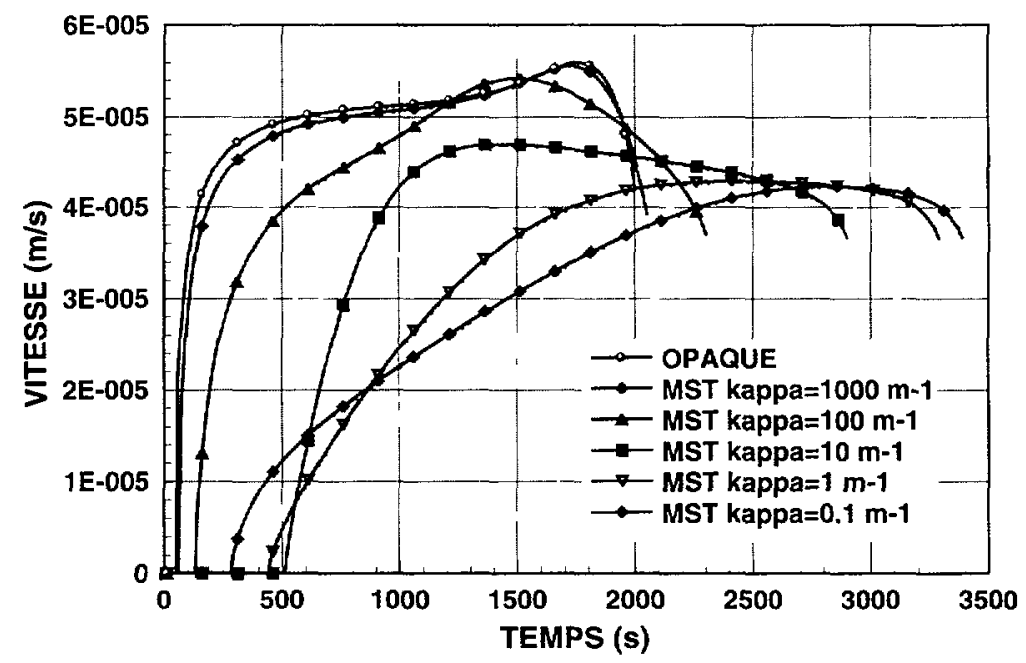

Fig. 8. - Évolution de la vitesse de fusion d'un mur plan gris de $10 \mathrm{~cm}$ en fonction du temps pour différents coefficients d'absorption.

[Evolution as a function of time of the melting velocity of a $10 \mathrm{~cm}$ gray wall for several absorption coefficients.]

Nous présentons des résultats pour les cas suivants :

1) la surface d'impact transparente et la surface froide noire (T-O),

2) les deux surfaces transparentes (T-T).

Les différentes profils de températures et indicatrices d'émission apparentes $\varepsilon(\varphi)=L / L^{\circ}$ pour les deux surfaces ont été reportées sur les figures $9 \mathrm{a}$, b et 10a, b. Le cas (T-O) présente un comportement totalement différent d'un mur à deux surfaces opaques, puisqu'au cours du temps la surface "froide" s'échauffe très rapidement, pour devenir plus chaude que la surface d'impact ; à partir d'un certain moment, la situation s'inverse. et après une phase de quasiisothermie du milieu, la surface d'impact voit sa température devenir supérieure à celle de la surface froide : le régime devient stationnaire au bout de 3956 secondes, avec une zone interne plus chaude que la surface d'impact, proche de la température de fusion alors que la fusion n est pas amorcée. Le dernier cas (T-T) présente un comportement singulier, puisqu'il apparait au bout de 736 secondes une fusion à l'intérieur du milieu, alors que la fusion de la surface dimpact n'a pas encore commencé ; les deux indicatrices d'émission ont alors des comportements très comparables à celui déjà évoqué.

Les indicatrices d'émission apparente, $\varepsilon(\varphi)=\frac{\pi L(\varphi)}{\sigma T_{\mathrm{f}}^{4}}$. définies par le rapport de la luminance directionnelle émergeant de la surface, soit immobile soit d'impact, sur la luminance de corps noir à la température $T_{f}$, prennent en compte le champ de température interne dans un MST. Sur les figures 10a, $b$, elles ont une structure homogène typique des indicatrices de plaque plane [12] ; seule leur intensité est modifiée au cours du temps, reflétant l'évolution des profils de température au cours de la fusion. 


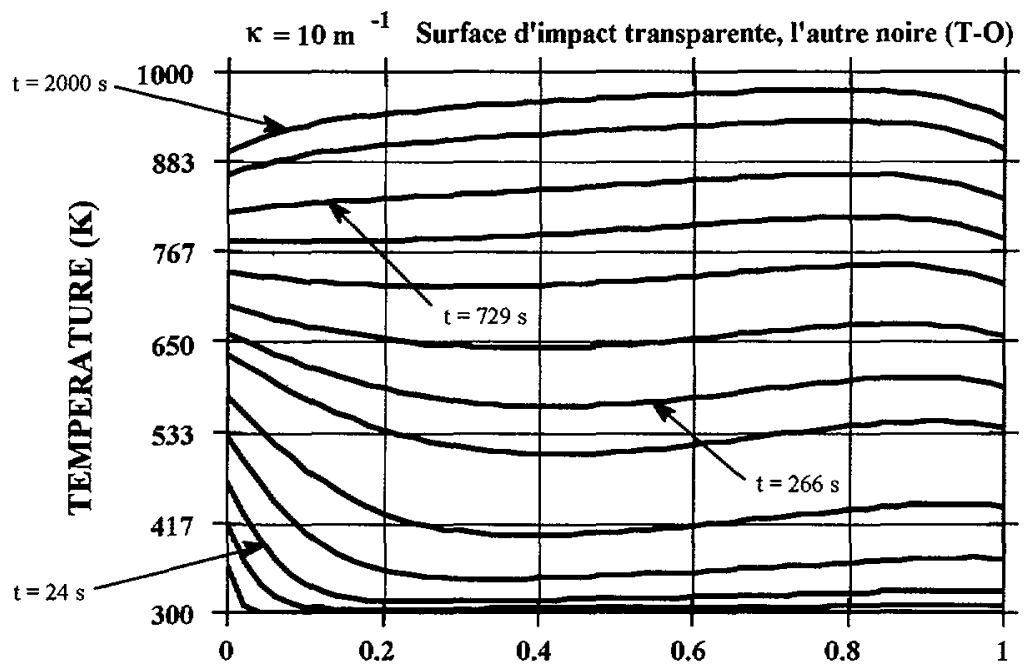

a)
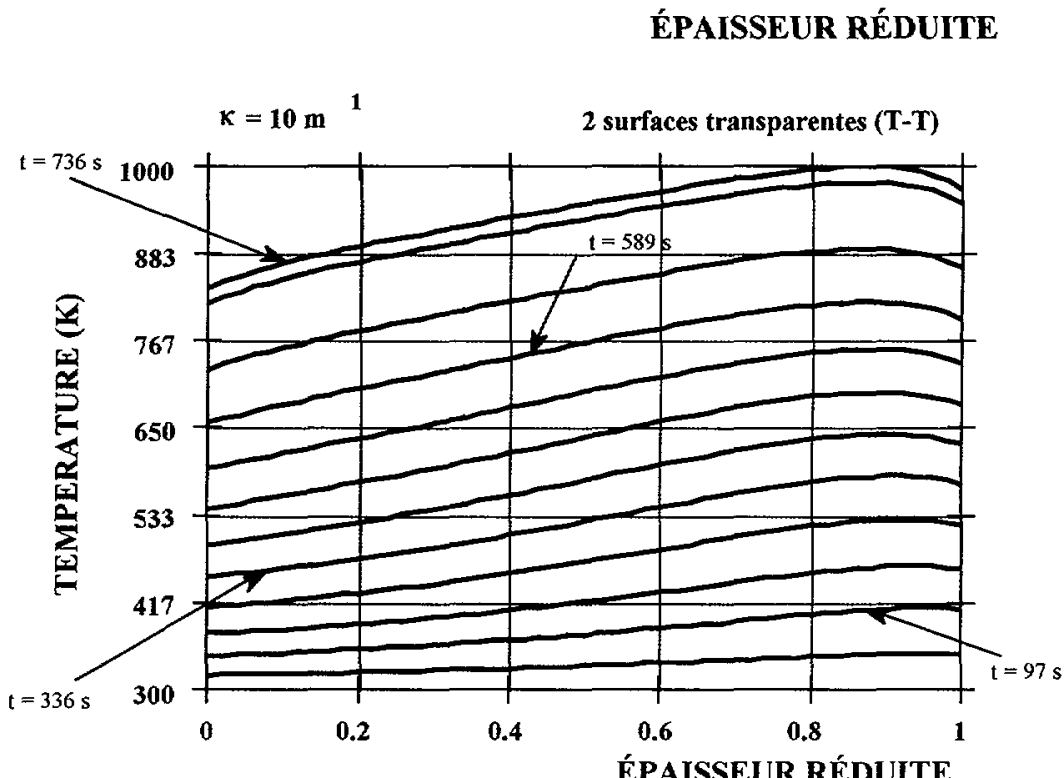

b)

ÉPAISSEUR RÉDUITE

Fig. 9. - Profils de température dans le mur de MST gris de coefficient d'absorption $10 \mathrm{~m}^{-1}$ à différents instants, a) surface d'impact transparente, surface fixe opaque noire, b) les deux interfaces transparentes.

[Temperature profiles of a $10 \mathrm{~m}^{-1}$ STM wall for several times, a) transparent impact surface, opaque fixed surface, b) the two boundaries are transparent.]

\section{Cas d'un milieu non-gris thermo-dépendant}

Pour un cristal, par exemple un halogènure alcalin, le coefficient d'absorption du milieu est constitué d'un fond continu dû aux interactions multi-phonons dans la région du bord d'absorption. Le coefficient d'absorption d'un cristal à la fréquence fondamentale peut s'exprimer sous 
2 interfaces transparentes: indicatrice de la surface froide $(T-T)$

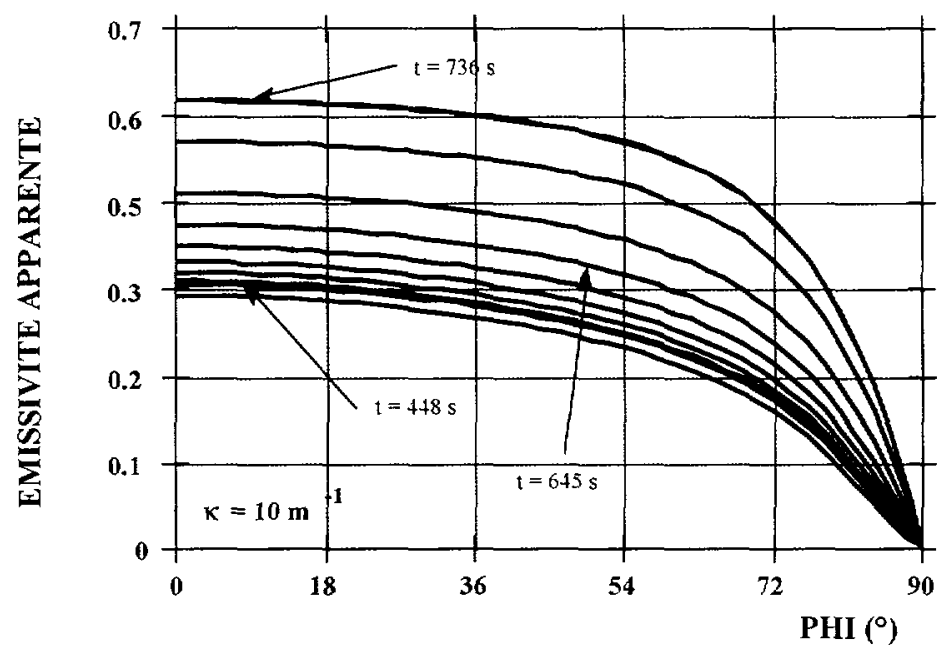

a)

2 surfaces transparentes: indicatrice de la surface d'impact (T-T)

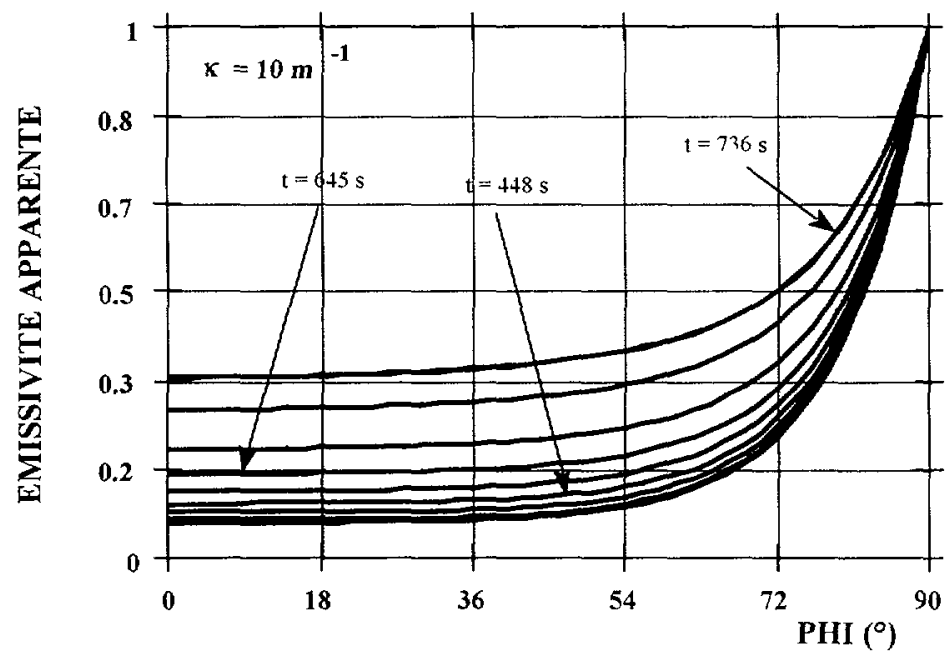

b)

Fig. 10. - Émissivité apparente d'un mur plan de coefficient d'absorption $10 \mathrm{~m}^{-1}$ avec ses deux frontières transparentes, a) pour la surface froide, b) pour la surface d'impact.

[Apparent emissivity of a $10 \mathrm{~m}^{-1}$ plane gray wall with two transparent boundaries, a) for the cold surface, b) for the impact surface.]

la forme $[7]$ :

$$
\kappa_{\nu}(T)=\kappa_{0}\left(\frac{e^{\frac{n \nu_{0}}{\hbar_{\mathrm{B}} T}}}{e^{\frac{\pi \nu_{0}}{h_{\mathrm{B}} T}}-1}\right)^{\frac{\nu}{\nu_{0}}-1} e^{-3 \frac{\nu}{\nu_{0}}}
$$

$\beta$ étant une constante caractéristique propre au cristal, $h$ la constante de Planck et $k_{\mathrm{B}}$ la constante de Boltzmann, la fréquence fondamentale $\nu_{0}$ variant avec la température par exemple 
pour le fluorure de lithium selon [13]

$$
\nu_{0}(T)=\nu_{0}(0) \exp \left(-3 \gamma_{\mathrm{G}} \int_{T^{\prime}=0}^{T} \alpha\left(T^{\prime}\right) \mathrm{d} T^{\prime}\right)
$$

avec $\gamma_{G}$ la constante de Gruneisen et $\alpha$ le coefficient de dilatation linéaire du cristal. Ainsi, le coefficient d'absorption du fluorure de lithium vaut $\kappa=1000 \mathrm{~m}^{-1}$ à un nombre d'ondes de $1000 \mathrm{~cm}^{-1}$ pour une température de $300 \mathrm{~K}$, alors que cette même valeur est atteinte à $1630 \mathrm{~cm}^{-1}$ pour une température de $1000 \mathrm{~K}[14]$ : on peut donc concevoir qu'une variation importante du coefficient d'absorption en fonction de la température modifie de façon conséquente le processus de fusion dans le problème de Stefan, particulièrement lorsque les deux interfaces sont transparentes et que le front de fusion se développe à l'intérieur du milieu ; mais dans un tel cas, il faudrait reformuler le problème.

Cependant, l'expression (32) peut être insérée dans notre modèle tant en utilisant la MOD que la LR, les deux techniques étant compatibles avec une thermo-dépendance du coefficient d'absorption, en considérant que dans chaque maille la température est constante, ainsi que le coefficient d'absorption : le processus de résolution comportera alors une itération supplémentaire recalculant à chaque fois le champ d'absorption. En outre, la thermo-dépendance du coefficient d'absorption n'est pas un obstacle au développement de la technique inverse exposée dans l'article suivant [1] pour retrouver la position du front de fusion.

\section{Conclusion}

Les modèles conducto-radiatifs développés dans le cadre du problème de la frontière mobile nous ont fourni des résultats sur le champ de température et la position du front de fusion dans un milieu semi-transparent. Ils ont permis de montrer d'une part, une très grande différence de comportement thermique entre le cas du mur opaque et le mur semi-transparent, et d'autre part, une influence notable de la transparence des frontières. Dans ce dernier cas l'existence d'une indicatrice d'émission apparente directionnelle tenant compte des particularités du champ thermique offre des informations supplémentaires permettant de contrôler l'état thermique interne du système. Une connaissance suffisamment précise du modèle direct permet d'envisager la reconstruction de la position du front de fusion à partir de données de mesures sur la température de paroi froide, indirectement influencée par l'avancée du front, cela grâce à l'utilisation de techniques inverses.

\section{Bibliographie}

[1] Yousefian F., Le Dez V. et Lallemand M., Problème de Stefan inverse dans un milieu semitransparent gris à parois noires, J. Phys. III 6 (1996) 391-401.

[2] Habib I.S., Solidification of semi-transparent materials by conduction and radiation, Int. J. Heat Mass Transfer 14 (1971) 2161-2164.

[3] Abrams M. et Viskanta R., The Effects of Radiative Heat Transfer Upon the Melting and Solidification of Semi-transparent Crystals, Trans. ASME (1974) 184-190.

[4] Chan S.H. et Hsu K.Y., Application of a Generalized phase change Model for Melting and Solidification of Materials With Internal Heat Generation, 19th Thermophysics Conference, Snowmass, Co, Paper No AIAA-84-1736 (1984). 
[5] Viskanta R. et Xigi Wu., Effect of radition on the melting of glass batch, Glastech. Ber. 56 (1983) 138-147.

[6] Landau H.G., Q. Appl. Math. 8 (1950) 81-94.

[7] Lipson H.G., Bendow B. et Mitra S.S., Temperature dependence of multiphonons absorption in fluorite crystals, Optical Properties of Highly Transparent Solids, S.S. Mitra, Ed. (Plenum, 1975) pp. 87-98.

[8] Lallemand M., Variation des constantes thermophysiques des matériaux semi-transparents avec la température, Application à la thermique, Rev. Gén. Therm. 264 (1983) 901-908.

[9] Furzeland R.M., A Comparative Study of Numerical Methods for Moving Boundary Problems, J. Inst. Math. Appl. 26 (1980) 411-429.

[10] Ozisik M.N., Heat conduction (John Wiley \& Sons, Inc, 1993).

[11] Le Dez V., Huclin J.C. et Lallemand M., Directional emissivities of semi-transparent media. Cases of dihedron, infinite cylinder and cone, Glastech. Ber. 66 (1993) 127-137.

[12] Le Dez V., Couplage Rayonnement-Conduction-Forme géométrique libre dans un matériau semi-transparent à haute température, Application à l'Étude des Instabilités Hydrodynamiques dans le formage des silicates (Thèse Université de Poitiers, 1993).

[13] Adams R.V., Phys. Chem. Glass. 2 (1961) 34-49.

[14] Barker A.J. et Wilkinson G.R., The high frequency tail of the lattice absorption spectra of simple crystals, Optical Properties of Highly Transparent Solids, S.S. Mitra, Ed. (Plenum, 1975) pp. 45-47. 